\title{
Intracameral phenylephrine and ketorolac injection (OMS302) for maintenance of intraoperative pupil diameter and reduction of postoperative pain in intraocular lens replacement with phacoemulsification [Corrigendum]
}

Lindstrom RL, Loden JC, Walters TR, et al. Clinical

Ophthalmology. 2014;8:1735-1744.

On page 1736, last line of the first paragraph in the Materials and methods section, "NCT01193127" should be

"NCT01579565". 